\title{
A Study on the Efficient NFC-based A Forgery Prevention Method
}

Eun Kim, Su Jin Lee, Hyun Joo Yoo, Jung Won Choi, Seong Jeen Kim, Min Soo Jung

\begin{abstract}
Today, scale of counterfeit goods market is growing continuously. Thus, the way to verify the legitimate product is required. Earlier authentication methods are difficult for the verification from the user side. In this paper, we propose an efficient method for the forgery prevention.
\end{abstract}

Keywords-Forgery Prevention, Authentication, NFC

\section{Introduction}

Nowadays, damage accidents that there are distributed to forge with a famous genuine things by malicious operator are increasing constantly. And also, side of product manufacturer, they produce authenticated product by applying like a barcode, RFID, Hologram and etc. However, users are difficult to recognize the legitimate product such that the only way. For this reason, users want to know the correct information about the product they are buying. Therefore, we believe that can perform to authenticate safely by using stored authentication information in the server of AuC. And the more convenience method may be used by using a smart phone and NFC technologies. Thus, we propose an efficient NFC-based a forgery prevention method. And this time, a NFC-equipped smart phone and the authentication application are authentication tools. And we design to store authentication information in physically strong USIM file system.

Eun Kim/Kyungnam University

Kyungnam University

Changwon-si

Su Jin Lee/Kyungnam University

Kyungnam University

Changwon-si

Hyun Joo Yoo/Kyunganm University

Kyunganm University

Chanwon-Si

Jung Won Choi/Kyungnam University

Kyungnam University

Changwon-si

Seong Jeen Kim/Kyungnam Univeristy

Kyungnam University

Changwon-si

Min Soo Jung/Kyungnam University

Kyungnam University

Changwon-si

\section{Related Work}

\section{A. NFC(Near Field Communication)}

The NFC performs the communication at the $13.56 \mathrm{MHz}$ frequency band with the RFID [1-3]. And a NFC-equipped device that supports following three modes : Initiator, Target, and Reader/Writer. And it may support Card Emulator[4]. Therefore, active communication is possible mutually.

\section{Proposed NFC-based Forgery Prevention Method}

NFC-based Forgery Prevention Method that is proposed in our paper is divided into the pre-registration, user registration and authentication process. Firstly, the description of used the terms in our proposed method is same as follows.

\section{TABLE 1}

Terminology

\begin{tabular}{cc}
\hline \hline Symbol & Description \\
\hline OG & Original Goods \\
SN & Secret Number \\
AuC & Certification Authority(Server) \\
$\mathrm{E}(\mathrm{)}$ & Encryption \\
$\mathrm{H}(\mathrm{)}$ & Hash Function \\
$\|$ & Concatenation \\
$\mathrm{Puk}$ & Public Key \\
prk & Private Key \\
$\mathrm{T}_{\mathrm{ID}}$ & Unique Identification Number of Tag \\
$\mathrm{P}_{\mathrm{ID}}$ & Unique Identification Number of Product \\
$\mathrm{P}_{\mathrm{NAME}}$ & Product Name \\
$\mathrm{P}_{\mathrm{DATE}}$ & Production Date \\
$\mathrm{P}_{\mathrm{CODE}}$ & Producer code \\
$\mathrm{P}_{\mathrm{SLAN}}$ & Provider Sign \\
\hline
\end{tabular}


Our proposed method includes a product(Luxury Goods), Smart Device(Phone) enabled NFC, as following Fig.1

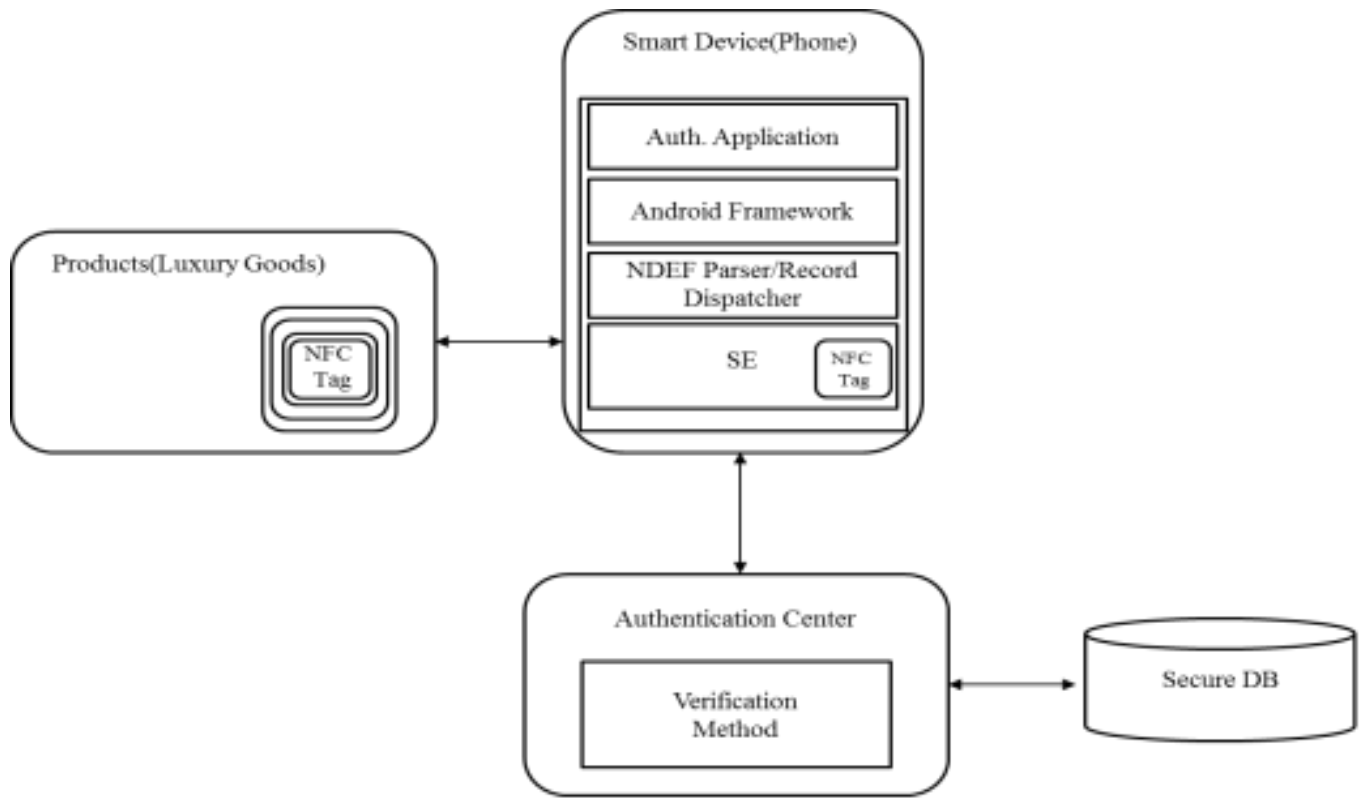

Fig.1 Architecture of the Authentication System

\section{A. Pre-Registration Phases}

This phase is performed before the product is shipped from the manufacturer. Firstly, manufacture writes various information like as $\mathrm{P}_{\mathrm{ID}}, \mathrm{P}_{\mathrm{NAME}}, \mathrm{P}_{\mathrm{DATE}}, \mathrm{P}_{\mathrm{CODE}}$ and $\mathrm{P}_{\mathrm{SLAN}}$. And also store same data to server of AuC. And this time, this is registration state that it is performed by manufacture, thus rewriting is impossible(ROM MASK).

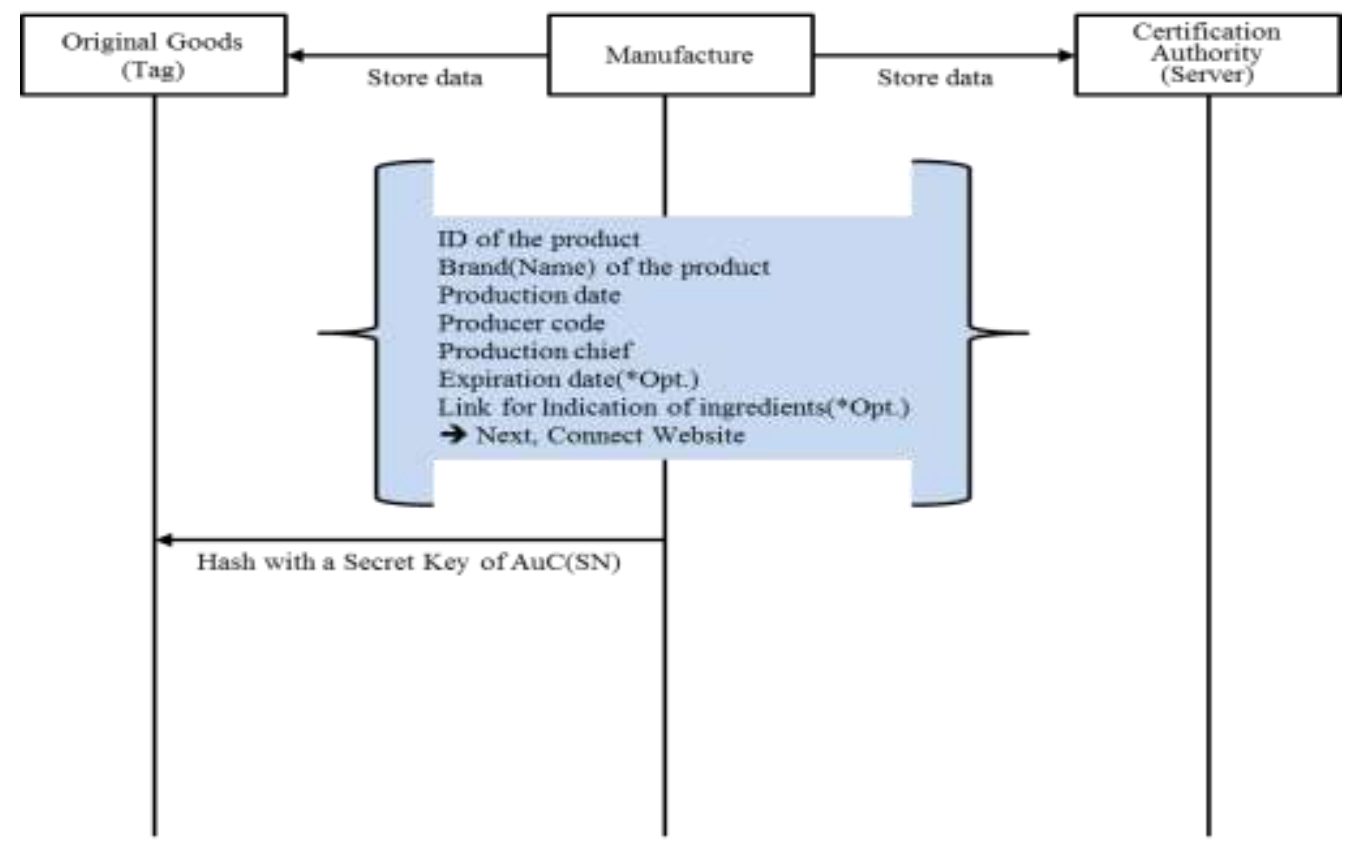

Fig.2 Pre-Registration Phase 


\section{B. User Registration Process}

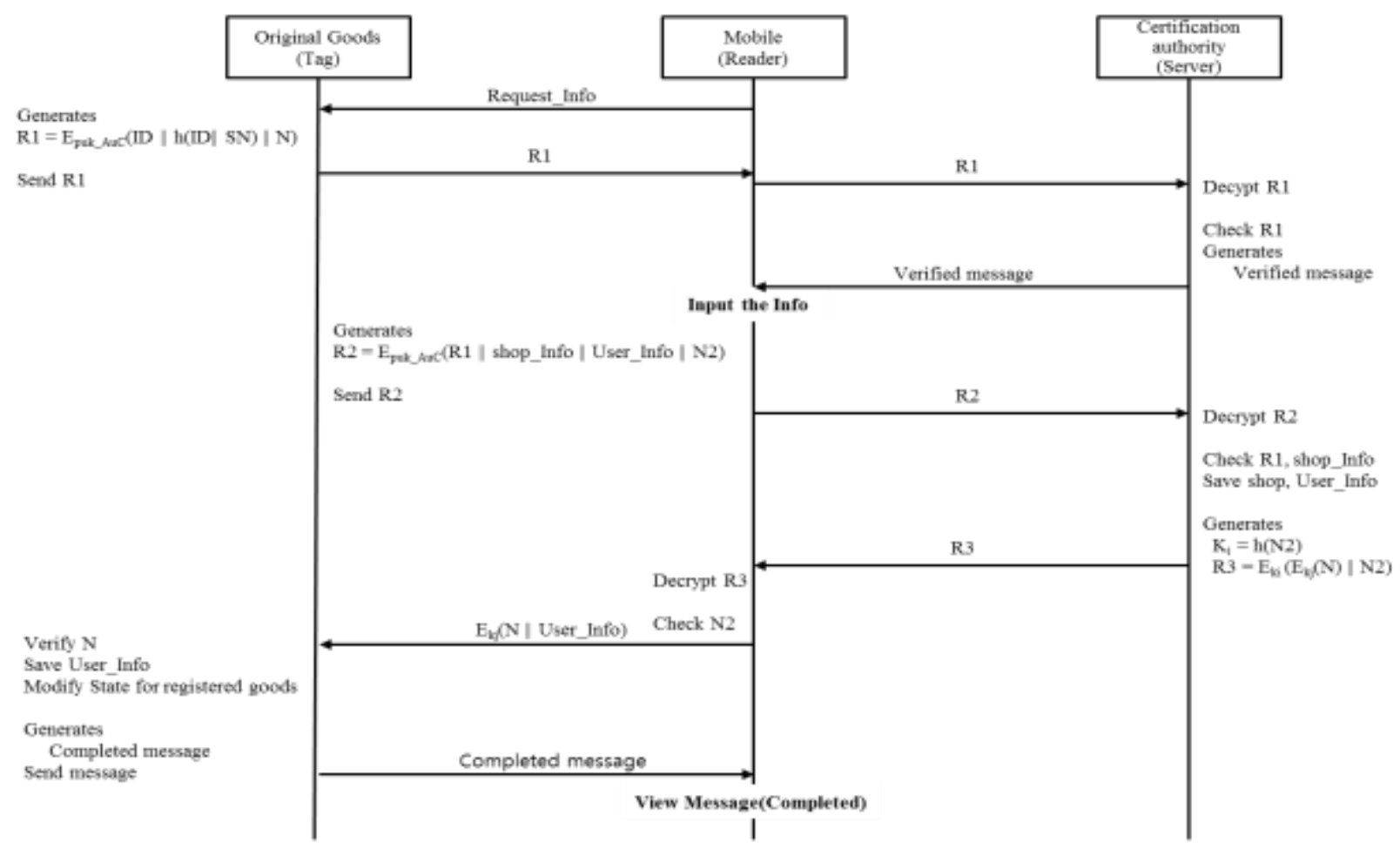

Fig.3 User Registration Process

This phase is processed when user purchases a product. The detailed step are as follows.

\section{- Scenario and Registration Step}

1. User tries to keep in touch with his mobile on the OG.

2. OG generates encrypted data R1 with nonce, $\mathrm{SN}$ of $\mathrm{AuC}$ and product ID using by public key of AuC. And sends R1 to mobile.

3. Mobile also sends R1 to AuC directly

4. AuC decrypts R1. And generates verified message, after checking the R1. And then, sends generated message to mobile.

5. Mobile display the result message.

6. Person who shop manager inputs the user and shop information through the App. And then, mobile encrypts the information of the product, shop and user with a nonce using by public key of AuC. And sends to $\mathrm{AuC}$ the generated message $\mathrm{R} 2$.

7. AuC checks R1(product ID), information of shop and user, after decrypting the R2. Make sure whether it stores in DB. And if verified, stores information of shop and user in DB.
8. Finally, generates R3 after generating key by nonce of mobile. Sends R3 to mobile.

9. Mobile decrypts the R3 using by public key of AuC. And then, checks generated a nonce value N2. Sends encrypted message with a nonce $\mathrm{N}$ and user information to OG.

10. OG stores user information, after verifying the nonce value $\mathrm{N}$. And modify state information as a registration finished product. Finally, sends it to mobile, after generating the completed message.

11. Mobile shows message it has been completed the registration.

\section{Authentication Process}

This phase is final process in our proposed method. And substantially, this is a step to verify the validity of the product. Through stored information in server of $\mathrm{AuC}$, to perform the verify about the product whether real or not. The detailed authentication processes are same as following. 
Proc. of the Intl. Conf. on Advances in Computing, Control and Networking - ACCN 2015.

Copyright (C) Institute of Research Engineers and Doctors, USA .All rights reserved.

ISBN: 978-1-63248-038-5 doi: 10.15224/ 978-1-63248-038-5-80

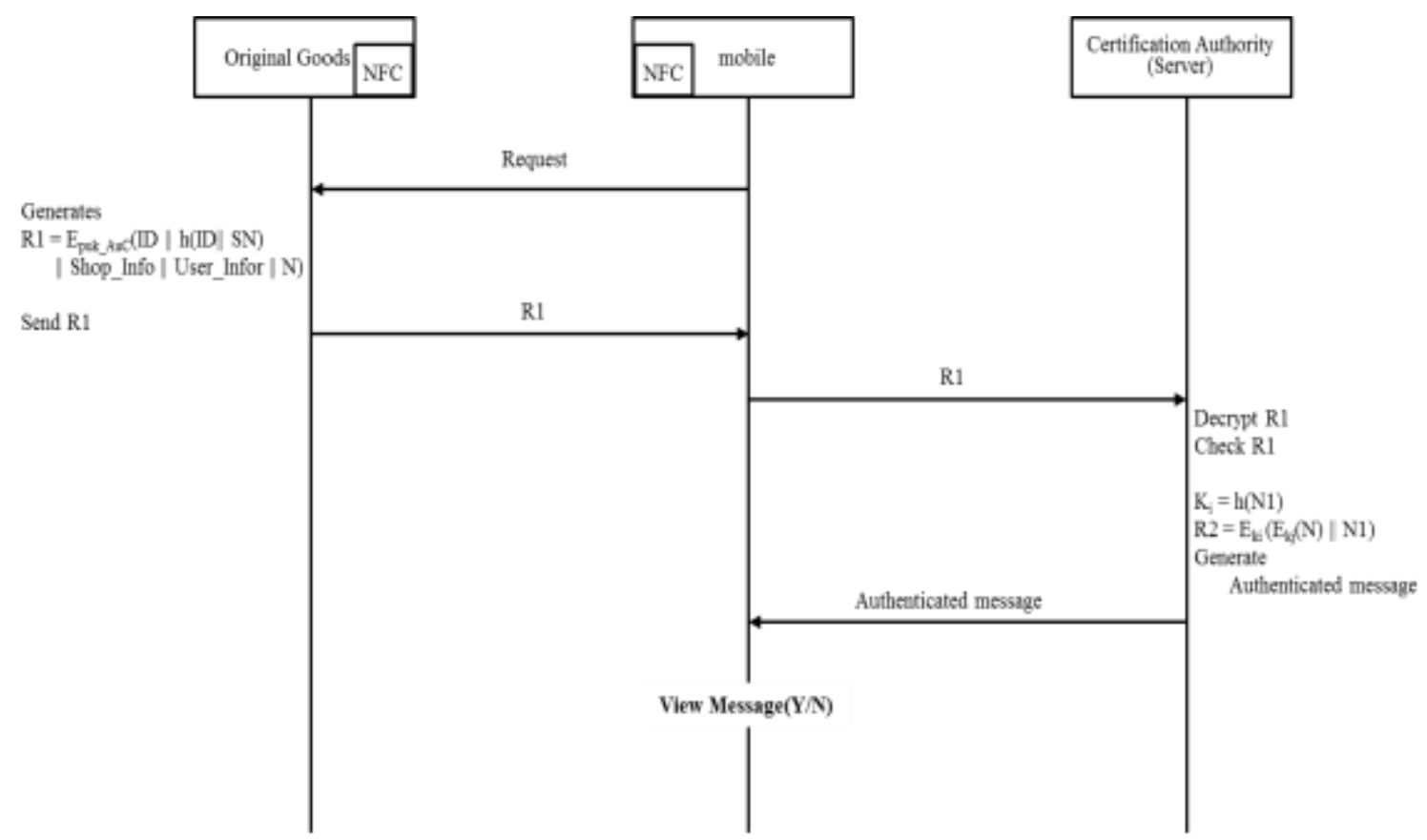

Fig.4 Authentication Process

\section{- Scenario and Authentication Step}

1. User tries to keep in touch with his mobile on the OG.

2. OG encrypts like as product ID, hashed message with a product ID and secure number SN of AuC, shop and user information, etc. using by a public key of $\mathrm{AuC}$. Thus, $\mathrm{R} 1$ is made and sent to mobile.

3. Mobile sends received data R1 to AuC.

4. AuC checks R1, after decrypting the R1 by own private key. AuC can get information of the product, shop and user. And then, verifies storing above data in DB. And finally sends it, after generating the message it has been completed the authentication.

5. Mobile shows message by own screen.

\section{Conclusion}

Our proposed authentication method for a forgery prevention can provide convenience to user. Because, user can directly access to product information using only a user's NFC-equipped smart phone. And because authentication information is stored in physically strong USIM file system, it can be stored and managed. Therefore, we propose an efficient NFC-based a forgery prevention method. Next, we will also process with the actual application and study based on this method.

\section{Acknowledgment}

This research was supported by Basic Science Research Program through the National Research Foundation of Korea(NRF) funded by the Ministry of Education, Science and Technology(2012R1A1B6002354).

This research was financially supported by the Ministry of Education, Science Technology (MEST) and National Research Foundation of Korea(NRF) through the Human Resource Training Project for Regional Innovation

\section{References}

[1] F. Michahelles, F. Thiesse, A. Schmidt and J.R Williams, "Pervasive RFID and Near Field Communication Technology," IEEE Pervasive Computing, Vol.6, No.3, 2007.

[2] M. Hutter and R. Toegl, "A Trusted Platform Module for Near Field Communication," in Conf. Rec. 2010 ICSNC Conf, France.

[3] H. C. Cheng, W. W. Liao, T. Y. Chi and S. Y. Wei, "A Secure and Practical Key Management Mechanism for NFC Read-Write Mode," in Proc. 13th ICACT Conf, Korea, 2011.

[4] NFC Activity Specification, NFCForum-TS-Activity-1.0, 2010.

[5] Eun Kim, Yun Seok Lee, Min Soo Jung, "Design and Implementation of an Authentication System for Anti-Forgery using the Smart Card," Journal of Korea Multimedia Society, Vol.14, No.2, pp.249-257, Feb.2011.

[6] ISO 18092 (ECMA-340). Information technology - Telecommunications and information exchange between systems - Near Field Communication - Interface and Protocol (NFCIP-1). Int. Organization for Standardization, Geneva, 2004.

[7] Moblie Near Field Communication (Mobile NFC) Stepping Stones, Vers ion 1.0.0, SIMalliance, pp.10-15, (http://www.simalliance.org).

[8] Hasoo Eun, Hoonjung Lee, Heekuck Oh, "Conditional Privacy Preserving Security Protocol for NFC Applications, ConsumerElectronics, IEEE Transactions on , vol.59, no.1, Feb. 2013. 
Proc. of the Intl. Conf. on Advances in Computing, Control and Networking - ACCN 2015.

Copyright (C) Institute of Research Engineers and Doctors, USA .All rights reserved.

ISBN: 978-1-63248-038-5 doi: 10.15224/ 978-1-63248-038-5-80

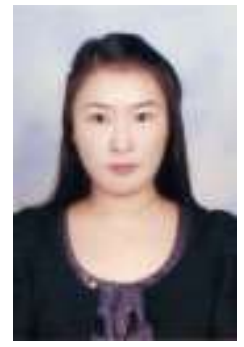

Eun Kim received the B.S and M.S. degree in computer engineering from Kyungnam University, Changwon, Korea, in 2009, 2011. She will receive PH.D in Feb. 2015. Her research interests include Java Card, Security, Authentication and Secure Protocol.

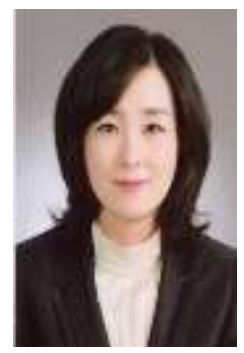

Su Jin Lee received the B.S and M.S. degree in computer engineering from Kyungnam University, Changwon, Korea, in 1998,2000 . She is working on a PH.D. Her research interests include Java Technology, Network Security.

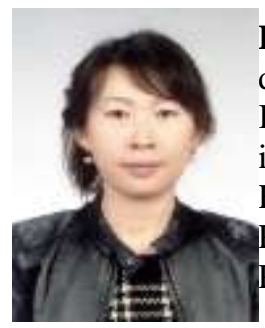

Hyun Joo Yoo received the B.S and M.S. degree in computer engineering from Kyungnam University, Changwon, Korea, in 1998, 2002. She is working on a PH.D. Her research interests include NFC, Mobile Payment Security, Mobile Platform Programming.

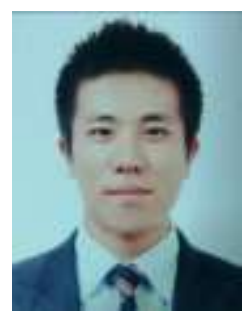

Jung Won Choi received the B.S degree in computer engineering from Kyungnam University, Changwon, Korea, in 2013. He will receive M.S degree in Feb.2015.

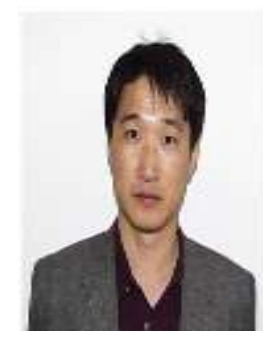

Seong Jeen Kim (S. J. Kim) received the B. S. degree, the M.S. degree and the Ph.D. in electrical engineering from Korea University in Seoul, Korea, in 1983, 1985 and 1993, respectively. For a while, he had studied in the Department of Electrical Engineering and Computer Science, University of Michigan, in An Arbor toward $\mathrm{Ph}$. D degree from 1988. Since 1994, he has worked as professor in the Department of Electronic Engineering, Kyungnam University in Korea. His research interests are in the areas of semiconductor materials and devices, especially related to sensors.

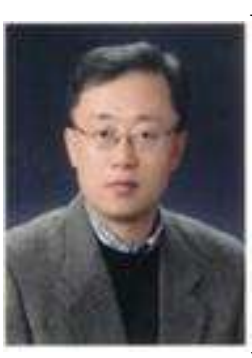

Min Soo Jung received the B.S degree in computer engineering from Seoul National University, Seoul, Korea, in 1986. And he received the M.S degree and the PH.D in electrical engineering from KIST(Korea Advanced Institute of Science and Technology)in 1988, 1994. Since 1990, he has worked as professor in the Department of Computer Engineering, Kyungnam University in Korea. His research interests include Java Technology, Java Machine, Tag Technologies related to Electroic Payment. 